\title{
Study of charge quantization in individual silicon quantum dots using Kelvin Probe Force Microscopy
}

\author{
M. A. Salem, Y. Tsuchiya, K. Usami, H. Mizuta* and S. Oda \\ Quantum Nanoelectronics Research Center, Tokyo Institute of Technology \\ *Department of Physical Electronics, Tokyo Institute of Technology \\ O-okayama 2-12-2, Meguro-ku, Tokyo 152-8552, Japan \\ Tel: +81-3-5734-3048, E-mail: soda@pe.titech.ac.jp
}

\section{Introduction}

Nanocrystalline silicon (nc-Si) dots have attracted a great interest due to their unique properties such as charge storage [1], and photoluminescence [2]. For studying structural and material properties of the individual nc-Si dots, scanning probe microscopy (SPM) techniques have widely been used, which facilitate the lateral and vertical resolutions of down to $0.1 \mathrm{~nm}$ and $0.01 \mathrm{~nm}$, respectively. In particular, the kelvin probe force microscopy (KFM) [3] is preferred when we need to observe the surface potential or work function profiles. Since the first attempt was reported by Nonnemacher et al [4], the KFM has been applied for investigating metal thin films [5], semiconductors [6] and nano particles [7]. The operating principle of the KFM is setting off the electrostatic forces between the tip and the sample by applying a feedback potential, this force at a frequency $\omega$ is given by

$F=\frac{d C}{2 d z}\left[\Delta \Phi-\left(V_{d c}+V_{a c} \sin (\omega t)\right)\right]^{2}$

where $\mathrm{z}$ is the tip-to-sample distance, $\mathrm{C}$ the capacitance, $\Delta \Phi$ the contact potential difference (CPD) between the tip material and the sample material, and $\mathrm{V}_{\mathrm{dc}}$ and $\mathrm{V}_{\mathrm{ac}}$ are the $\mathrm{DC}$ and $\mathrm{AC}$ components of the applied voltage, respectively. Due to the applied AC voltage, the cantilever no longer vibrates mechanically but electrically. The electrostatic force at frequency $\omega$ is given as:

$F_{\omega}=\frac{\partial C}{\partial z} V_{a c} \sin (\omega t)\left(V_{d c}-\Delta \Phi\right)$

The amplitude of cantilever vibration will be directly proportional to the spectral component of the force if $\omega$ is close to the resonance frequency of the cantilever. Furthermore, if the $\mathrm{V}_{\mathrm{dc}}$ equals the CPD, $\Delta \Phi$, the force at $\omega$ would then be nullified. Recording the DC voltage with scanning the entire sample area therefore, enables mapping the surface potential of the sample on the nanometer scale.

\section{Experimental}

For preparing nc-Si dots, a thin $\mathrm{SiO}_{2}$ layer was grown using chemical oxidation of $\mathrm{H}_{2} \mathrm{SO}_{4} / \mathrm{H}_{2} \mathrm{O}_{2}$ on a p-type (100) Si substrate, and nc-Si dots about $2 \mathrm{~nm}$ to $8 \mathrm{~nm}$ in diameter were then deposited with VHF plasma decomposition of $\mathrm{SiH}_{4}$ described in details elsewhere [8].

Topographic and potential measurements were performed simultaneously under the normal ambient conditions using an AFM based Kelvin Force probe microscope using a rhodium coated silicon cantilever having the resonance frequency of about $24 \mathrm{KHz}$ and the force constant of $1.8 \mathrm{~N} / \mathrm{m}$. The area under investigation is $0.6 \mu \mathrm{m}$ x $0.6 \mu \mathrm{m}$ scanned with AC voltage $10 \mathrm{~V}$.

Charge injection into the $\mathrm{Si}$ dots was carried out using the biased tip in the contact mode. After positioning the conducting tip to the targeted point, the tip was biased at -5 $\mathrm{V}$ and was then edged nearer to the surface and kept in contact for 30 seconds. Then a non-contact mode KFM scan was performed, the change in the measured CPD of the dots upon charging indicates directly the charging energy added to each dot. Further details on the charging process using the AFM tip as well as a theoretical model for estimating the charge transferred can be found elsewhere [9]. Figure 1 shows the experimental flow of the measurement.

\section{Results and discussion}

Figure 2 shows the simultaneously scanned topography (a) and potential (b) images. Note that the lateral dot dimensions are overestimated as the AFM image is given as convolution of the sample and tip shapes. However, we may obtain the diameter of the individual dots much more precisely from the vertical topography data, the dot diameter ranges from about 2 to $8 \mathrm{~nm}$.

The potential image is shown in Fig.2 (b), where the clear distinction between the $\mathrm{Si}$ dots and the substrate is seen. In addition, small and large dots are clearly distinguished in the potential distribution, and we found that the larger the dots the lower the CPDs. Despite a variety of past KFM studies our present work features the first direct measurement of the size-dependent potential of the silicon quantum dots although the obtained CPDs can be affected by the existence of any adsorption layers on the surface [10]. Figures 3(a) and (b) show the images obtained after charging. As expected the topographic height did not show any significant change after the charging since the KFM measurement system cancels the electrostatic forces between the tip and the sample. Therefore charging the sample has no effects on the sample topography obtained with the KFM. In that sense the KFM is different from other non-contact or tapping mode measurements, in which the topographic images are indeed affected by all types of forces, and the charge transferred from tip to sample is estimated only in an indirect manner, in which the topographic height change (or apparent height) is analyzed $[9,11]$.

The change in the dot spatial distribution after the charging is attributable to the repulsive forces between the charged dots. It is interesting to see that larger dots show the higher potential than that for smaller dots while the substrate remains at almost the same potential of about $300 \mathrm{mV}$. This 
manifests that only the Si dots were charged and that the KFM facilitates to detect the size dependence of the charge confined in the nanostructures experimentally.

The number of electrons injected into each dot can be estimated by comparing the measured CPD change with the charging energy, $E_{c}=(n e)^{2} / C_{d o t}$, calculated for the Si dots where $C_{d o t}$ is dot capacitance and $\mathrm{n}$ is the number of injected electrons. Figure 4 plots the dot CPD change as a function of the dot diameter, along with the charging energy calculated with one, two and three extra electrons per dot. This shows that only one electron is stored in the nc-Si dot with diameter up to $2.8 \mathrm{~nm}$, and three electrons are stored in the dots with diameter in the range from $4.7 \mathrm{~nm}$ to $7.4 \mathrm{~nm}$. The dot with diameter of $4.6 \mathrm{~nm}$ likely stores two electrons although this is not very clear as we did not find the dots in the range of $2.8 \mathrm{~nm}$ to $4.7 \mathrm{~nm}$ in the present sample.

\section{Conclusion}

In conclusion, a simple, reliable and precise method of charging nc-Si dots as well as probing the charge injected into the dots has been presented based on the simultaneous observation of the KFM topography and the CPD change of the dots. The number of injected electrons has been identified by comparing the measured CPD changes as a function of the dot diameter with the charging energy calculated for various numbers of electrons. This method may provide a powerful tool for investigating local electronic states and transport properties in the modern nanoelectronic devices.

\section{Acknowledgment}

This work has been supported by CREST program of Japan Science and Technology Agency (JST).

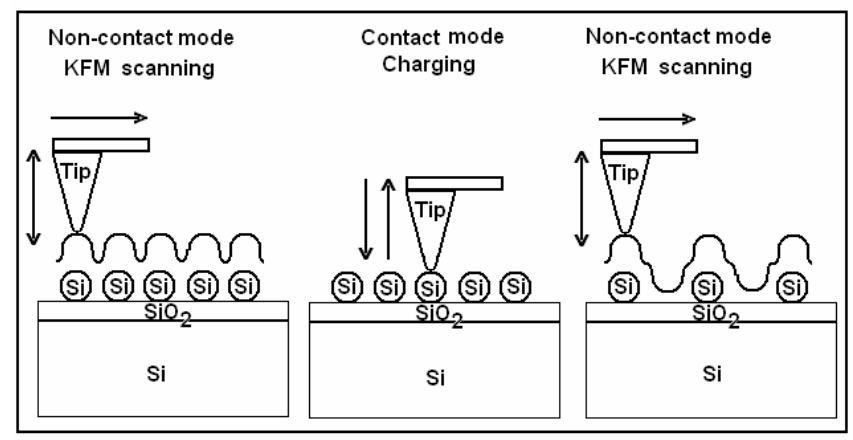

Fig. 1 The Experimental flow of the measurement process.

\section{References}

[1] B. J. Hinds, T. Yamanaka, and S. Oda, J. Appl. Phys. 90, (2001) 6402.

[2] K. Arai and S. Oda, Physica Status Solidi(c) 0 (2003) 1254.

[3] N. Shimizu, M. Ikeda, E. Yoshida, S. Miyazaki and M. Hirose, Jpn. J. Appl. Phys. 39 (2000) 2318.

[4] M. Nonnenmacher, M. P. O'Boyel, and H. K. Wickramasinghe, Appl. Phys. Lett. 58 (1991) 2921.

[5] M. P. O'Boyel, T. T. Hwang, and H. K. Wickramasinghe, Appl. Phys. Lett. 74 (1999) 2641.

[6] S. Kitamura, K. Suzuki, M. Iwatsuki and C. B. Mooney, Appl. Surf. Sci. 157 (2000) 222.
[7] S. Ono, M. Takeuchi and T. Takahashi, Ultramicroscopy, 91 (2002) 127.

[8] T. Ifuku, M. Otobe, A. Itoh, and S. Oda, Jpn. J. Appl. Phys. 36 (1997) 4031.

[9] S. Banerjee, M. A. Salem and S. Oda, Appl. Phys. Lett. 83, (2003) 3788 .

[10] B. Bhushan, A. Goldade, Wear, 244 (2000) 104.

[11] E. A. Boer, L. D. Bell, M. L. Brongersma and H. A. Atwater, J. Appl. Phys. 90 (2001) 2764.

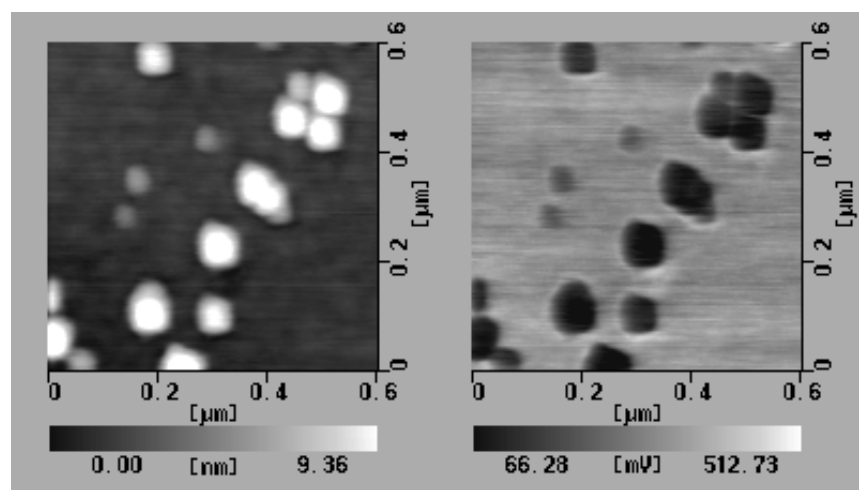

Fig. 2 Simultaneous topographic (a) and potential (b) KFM images for the nc-Si dots before charging.

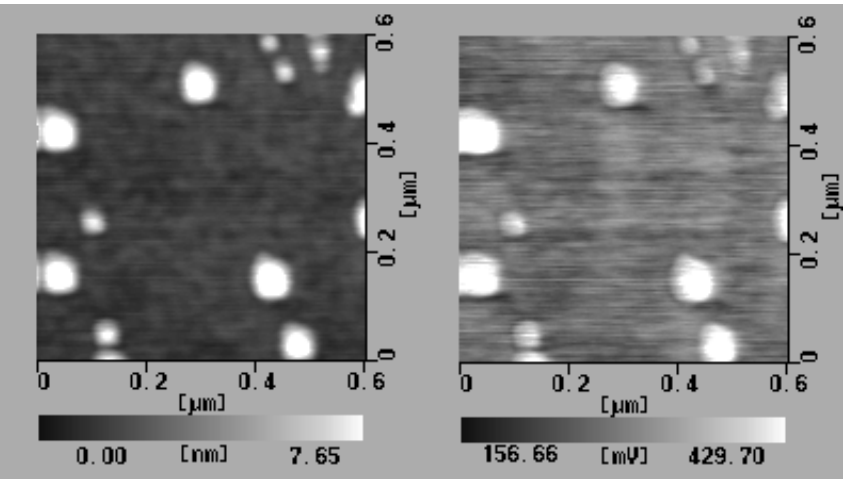

Fig. 3 Simultaneous topographic (a) and potential (b) KFM images for the nc-Si dots after charging.

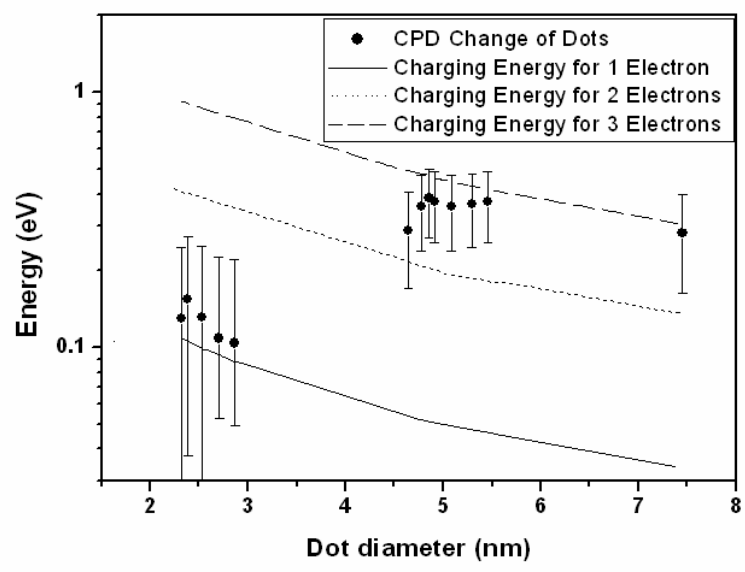

Fig. 4 The change in the CPD for the nc-Si dots with various diameter (solid circles) and the calculated charging energy as a function of the dot diameter with the number of extra electrons on the dot of one (a thin solid line), two (a dotted line) and three (a broken line). 